\title{
Virtual supervision of pharmacy undergraduate research projects during the COVID-19 lockdown in Zimbabwe
}

\author{
C.M.J. Matyanga*, B. Dzingirai, T.G. Monera-Penduka \\ School of Pharmacy, College of Health Sciences, University of Zimbabwe, Zimbabwe
}

\author{
Keywords \\ Pharmacy \\ Research \\ COVID-19 \\ Supervision \\ Zimbabwe
}

\section{${ }^{*}$ Corresponding author: celiammi@yahoo.com}

\begin{abstract}
Summary: The coronavirus 2019 (COVID-19) pandemic has led to national lockdowns in multiple states. The education system, including pharmacy education, has been negatively affected by the subsequent closure of universities. Many institutions have resorted to virtual learning, which work best in settings with fast, stable internet connections and where electricity is readily available. In resource-limited settings, popular virtual interaction platforms like Zoom may not work, and educators have to devise novel ways to continue teaching. The University of Zimbabwe used innovative mobile phone-based processes to ensure continuation of the final-year pharmacy students' honours research projects, supervision, and timely submission. This facilitated rapid communication between students and professors and allowed effective guidance for the students' research process. All students completed their projects; 13 out of 16 submitted their write-ups within the deadline; all students passed, $69 \%$ scored an upper second (2.1) grade or better, indicating the high quality of the majority of the research projects.
\end{abstract}

\section{Background and Context}

The coronavirus 2019 (COVID-19) pandemic has led to worldwide travel restrictions and national lockdowns. The education system, including pharmacy education, has been negatively affected by the subsequent closure of universities (Brazeau, 2020). Many institutions have resorted to virtual learning which work best in settings where there is a fast, stable internet connection and where electricity is readily available. In resource-limited settings, where those are not available, popular virtual interaction platforms like Zoom, Microsoft Teams and Google Classroom may not work, and educators therefore have to devise novel ways to continue teaching (Okereke et al., 2020). The University of Zimbabwe, School of Pharmacy was closed on the 23rd March 2020 due to COVID-19 lockdown restrictions (Mukeredzi, 2020). The closure of the School affected the undergraduate final-year students who were due to finish their data collection and write up of their honours research project reports. These students conducted a research project in their final year, which is a requirement for graduation with an honour's degree.
Some private universities in Zimbabwe have been conducting virtual lessons via Moodle, Zoom, Microsoft Teams, Google Classroom and emails. These methods are effective when there is a stable internet connection. For public universities, both lecturers and students have limited resources, and the majority face challenges with acquiring individual electronic gadgets, out-of-pocket spending on internet data bundles, lack of constant power supply and poor and unstable internet connection to facilitate virtual engagement.

\section{Educational Description}

The undergraduate pharmacy students were expected to complete data collections and compile research project write-ups during the period coinciding with the COVID-19 lockdown. Submission of the project write-ups had a set deadline. Project supervision included the lecturer receiving and reviewing the project results and the write-up documents. The traditional ways of supervising, which include face-to-face meetings and emails, were not feasible during the COVID-19 induced lockdown. Having observed 
that students communicated important learning-related information over self-organised WhatsApp groups during the course of the year, faculty conducted a small poll to determine the accessibility of WhatsApp and willingness to use the platform for research project-related communication. All students under the faculty's supervision were agreeable.

WhatsApp is a mobile-based messaging platform, which allows users to send texts and voice messages instantly. Traditionally, WhatsApp is used as a social media platform for non-official communications. It enables voice and video calls, sharing images, documents, and other media (Whatsapp, 2020). It uses very little data and the major mobile network providers in Zimbabwe run promotions for bulk data purchases that students often take advantage of. The platform supports the uploading of various types of document formats such as files in portable document format (PDF) and slideshows, voice notes and internet-based calls (Church \& De Oliveira, 2013).

All 16 students being supervised had smartphones that were capable of hosting the WhatsApp application. The data requirements for WhatsApp are lower than other online learning platforms like Zoom or Google Classroom. The University faculty, therefore, proceeded to form small WhatsApp groups, each with a lecturer supervising the students and used the platform for communication.

Sections of the project write-ups that the faculty expected to be written up at each stage were shared with the supervisor through the WhatsApp group as was any feedback for improvement. The feedback included guidance on methods, data analysis, discussion and presentation of the project report. Any feedback that was thought to be of a sensitive nature or was particularly relevant to any one student was given in a direct message to the individual student. In cases where longer descriptions and clarifications were required, the supervisor used the voice note and web calling function of the WhatsApp platform. The outcomes for the evaluation of the project supervision were students submitted the project write-up on time, sat the oral project examination and the quality of the research project as reflected by the marks obtained.

\section{Outcomes and Recommendations}

The option of using the WhatsApp platform for teaching and reviewing pharmacy undergraduate students' projects allowed fast communication and effectively guided the students' research process. In addition, the ability to upload files in PDF and PowerPoint formats that the department use to teach research methods provided additional reference materials to guide the students' research process. The ability to see which students were online facilitated direct real-time communication for individual corrections. The voice call and video calling support assisted in making follow-ups. As a result, all 16 students managed to complete their data collection, analysis and project write-ups. All students sat for the oral examination and 13 out of 16 submitted by the deadline. All students passed, with $69 \%$ scoring at least an upper second (2.1) grade or better, indicating the good quality of the majority of the research projects. Using WhatsApp may be the best option for teaching small numbers in resource-limited settings like Zimbabwe.

\section{References}

Brazeau, G.A., (2020). Lessons Learned and Brighter Opportunities for Pharmacy Education Amid COVID-19. American Journal of Pharmaceutical Education, 84(6). https://doi.org/10.5688/ajpe8230

Church, K., \& De Oliveira, R. (2013). What's up with WhatsApp? Comparing mobile instant messaging behaviors with traditional SMS. In Proceedings of the 15th international conference on Humancomputer interaction with mobile devices and services, 352-361. https://doi.org/10.1145/2493190.2493225

Mukeredzi, T. (2020). Under pressure, government closes universities and schools. Available at: https://www.university worldnews.com/post.php?story=20200323065236567

Okereke, M., Williams, A.E., Emmanuella, N.C., Ashinedu, N.U., \& Mairaj, M.W. (2020). COVID-19: challenges affecting the uptake of elearning in pharmacy education in Africa. Pan African Medical Journal, 35(2), 70. https://doi.org/10.11604/pamj.supp.2020.35.2.23910

Whatsapp. (2020). Simple. Secure. Reliable messaging. Available at: https://www.whatsapp.com/features/ 